\title{
Space Are Places in Which We Learn
}

\author{
Thomas Kvan
}

\begin{abstract}
We make and occupy spaces for purposeful activities. Significant investments are made in learning spaces in schools without adequate consideration of pedagogical and architectural issues. This chapter notes that it is a broad and multifaceted challenge to guide capital investments that deliver better learning outcomes in schools. It introduces the research presented in this volume and some of the underlying concepts and considerations embedded in the work of LEaRN and the projects described in following chapters.
\end{abstract}

At the start of the twentieth century, John Dewey published great insights into the state of education and the experience of children in schools (Dewey 2001). In particular he wrote of the benefits of organising learning around activity and purpose and that the school rooms (as he called them) needed to provide for active occupation. He observed that the rooms he knew necessitated behaviours of the children that were not conducive to that mode of learning, instead promoting passivity and thus disengagement. The obvious connection between the design of places of learning, schools and their outcomes has been widely engaged from both design and pedagogical perspectives. $^{1}$

The work of our research network, $\mathrm{LEaRN},{ }^{2}$ was initiated at a time when considerable financial capital was being directed to extending school facilities in our local community, that of the state of Victoria in Australia. You can find similar programmes of building and refurbishing schools across the world at various times, and unfortunately the converse, periods of neglect. Communities and governments appear to act periodically, responding to such factors as demographic shifts, reprioritisation

\footnotetext{
${ }^{1}$ For example, Sanoff, H. (1994). School Design. John Wiley \& Sons, Inc., 605 Third Avenue, New York, NY 10158, and Uline, C. L., \& Tanner, C. K. (2009). Effects of school design on student outcomes. Journal of Educational Administration.

${ }^{2}$ More information on the learning environments applied research network can be found online at https://research.unimelb.edu.au/learnetwork/home (accessed 10 December 2019).
}

T. Kvan (殴

The University of Melbourne, Melbourne, VIC, Australia

e-mail: tkvan@unimelb.edu.au

W. Imms and T. Kvan (eds.), Teacher Transition into Innovative Learning Environments, https://doi.org/10.1007/978-981-15-7497-9_1 
of budgets, political manifestos or as a capital stimulus for a faltering economy or perhaps simply shame. The investment in Victoria (a State of Australia) in 2008 was prompted by several such factors and was accompanied by questions of how to make such an investment effective for the declared purpose, that of learning.

The challenge of identifying if the investments have been effective is a complex one, as illustrated by the range of projects undertaken by our research network. All of these research projects have enquired into the large question of how to ensure that capital investments are well used by considering the diverse aspects of the question, drawing on the knowledge, ways of enquiring (sometimes called ways of knowing), methodologies and assumptions of different disciplines. The editors and authors in this book reflect the value of such diversity; my co-editor, Wesley Imms, draws on his deep knowledge of pedagogy and I from my experience in design.

Gaining strength from this diverse multidisciplinary background, projects in LEaRN have considered learning environments from a range of perspectives. We might summarise these as what we do, how we do it and how do we know what we have done. Some projects have examined construction techniques to understand if the most sustainable and cost-effective means are deployed to enable rapid response to changes in education demand. Other projects addressed the need for robust assessment criteria and methods for completed projects so that the lessons learned can be cycled back into the future school building. We have been commissioned to write guidelines for such future projects and we have assisted schools to use their facilities to achieve better learning outcomes.

Other projects have taken a more pedagogical perspective across different stages of learning, extending to examining the learning of students in primary up to tertiary systems, including particular cohorts such as special needs students or medical students on the wards. Recognising that schools serve broader purposes beyond the tasks of learning; we are also looking at how the sites can better serve as community hubs. All the projects are conducted collaboratively with partner schools, school districts, designers, industry suppliers and communities, with academic researchers taking the lead to organise and run the research.

The Innovative Learning Environments and Teacher Change (ILETC) project, which is the formal context from which this book emerges, is one project within this portfolio of projects. It was framed specifically at how we can help teachers to use the untapped potential of Innovative Learning Environments (ILEs) to improve learning outcomes for students. In particular the project wished to probe the contentious questions as to whether there is a link between quality teaching and effective use of the spaces in which they teach. In answering that question, the project sought to develop practical tools to assist teachers to adapt their teaching practices to maximise deeper learning.

The unspoken assumption in the work presented in this book is that we are all describing a place in which learning takes place. The participants, whatever their roles, congregate in one location to engage collectively in the activities, and the place is developed to facilitate these activities. We use resources to invest the site with features, facilities and meaning that enable the outcomes to be realised. We make these places, the schools, into sites where we can learn. We also know that 
this is not a work that can be completed, every insight brings opportunity and new questions. It is action research that informs work in progress. As such, we publish what we know, incomplete and unresolved. This book reflects a state of knowledge and opportunities in the next steps.

\section{It's Where We Are}

Instinctively, we all care about the spaces in which we conduct our daily lives. We seek out cafés that we find comfortable, we prefer certain parks or gardens when looking to relax, we complain to co-workers about the offices we are assigned, we spend our resources to make homes that respond to our needs. In all of these we are making an implicit assumption that space affects the way we behave and think, whether relaxing or concentrating, whether conducting mundane tasks or creative invention, whether we are learning or performing rote actions.

These instinctual decisions recognise that the spatial setting of our activity has some influence on our performance, that we relax more effectively in one place than in another or learn better in one school than another. This tacit understanding is often illustrated by a quote from Winston Churchill in 1943, 'We shape our buildings, and afterwards our buildings shape us' (United Kingdom 1943). In that debate about the proposed reconstruction of the House of Commons that had been severely damaged by German bombs in 1941, Churchill argued that the narrow rectilinear form of the chamber forced debaters to take clear positions, unlike a semi-circular space that facilitated subtle nuances by displacements along an arc. He argued too that there should be fewer seats than members so that there were no reserved seats, members had to respond to circumstances in their seating arrangements and hence could not retreat behind convention but could engage in spontaneous conversation. At key moments, the space would be overcrowded, the overcrowding itself adding urgency to the debate underway. In these comments, Churchill identified that the theatre of a space, its habitation, was a key and that the way the space affected the community engaged within it was an essential component to the particular practice of British parliamentary democracy.

\section{It's the Way We Act}

The connection that Churchill had identified is that the act of gathering to engage in a purpose is affected by the space in so far as the way the members of the particular community disport and participate is influenced by the geometry. In his argument, he addressed the conventions, cultures and conveniences, connecting these to the location in which they were enacted. Specifically, he was arguing for the reconstruction of a narrow debating chamber too small for the number of members and thus not meeting our contemporary understanding of 'functional space' but reinterpreting 
'functionality' to consider behaviours that may emerge because of the particularities of the space, its crowdedness, the narrowness. His argument was that our behaviours in the space make us, not the space itself. This is indeed the perspective we take, that the users of the spaces are in control, they make decisions about the use of the space and the experiences within.

\section{What Is It About the Space?}

The influence of the space on behaviour may be considered in a formal sense-if we are in a library, we behave as we should by some sense of convention of that library. It may be the semiotics of the spaces that bring behavioural change about-we can be conditioned to read the sign Library and react by lowering our voices and adopt other behavioural norms.

In effecting such a reaction, we have relied on our sight to read the sign and interpret this to behaviour. Our eyes can inform us of intentions through colour choices, sharp delineations between contrasting colours noting purposeful boundaries, or shifts from primary colours to pastels to indicate more subtle changes.

Other human senses can also be conduits for the message. An auditory threshold between a 'bright' ${ }^{3}$ space and a more muted one can lead to a tacit reaction of dropping the volume of your voice and adjusting action such as adjusting your pace.

Each of our senses can be engaged in this way. Bakeries that vent onto the street the smells of freshly made goods encourage us to linger or move on, as can sound and light qualities in other contexts.

While such factors are considered in the design of learning spaces, there are often more subtle factors than those registered by our primary senses. Several papers in this volume have explored the implicit enablers of spatial engagement, the affordances of a space.

\section{It's Our Choice}

While Dewey did not write explicitly about innovations in classroom design, his ideas have been influential in the emergence of other forms of learning environments and classroom designs. As Cuffaro (1995) notes, 'within a Deweyian framework the classroom environment demands primary attention'. She notes that few teachers will ever participate in the design process for new facilities, typically finding themselves assigned to an existing space and 'dealing with the unyieldingness of plumbing features and doorways'. She summarises with the observation that the important

\footnotetext{
${ }^{3}$ A term used to describe a space that has surfaces that reflect noise readily, privileging noises in the upper registers, so the space comes to be filled with competing sharp sounds.
} 
contribution of the teacher is in 'the choices we make, the physical and social arrangements we create'.

A common misunderstanding of Churchill's statement is that architectural design is deterministic, that space can cause an outcome. While we might respond to spaces by lowering our voice or moving slower, the effect is not determined, it is interpreted and suggested. The human actor in the space determines how they will behave. In summary, whether we respond to the opportunity offered by a particular space or collection of spaces is our choice, individually and collectively.

Our response can be to physical properties of the spaces, such as the light or sound, but also how we act or perform within the space. In all these the geometry of space and surface properties will influence us. Transparent walls connect our space to others; transformable or moveable items, furniture, walls, lights, allow us to control the experience. We can engage with space actively by using these properties, or passively and continue as ever.

What we do within the space is also to be considered. In daily life, we adapt our actions to a setting and in others we use the setting to enhance our actions. The act of teaching is not immutable; even the most seasoned of teachers are constantly reacting and responding to changes in demography, curricula, popular culture and professional insights. If we perceive an opportunity in a room, we use it. When the physical space no longer suffices, we adapt, we workaround, we hack.

Our reaction though is a choice and with that we consequently take on responsibility. The decision we make is driven primarily by the intention; whatever our role, we are in the place to help students learn. The goal is an alignment for purpose and outcome. There are multiple guides to our decisions such as prior experience; tacit and conveyed knowledge; cultural assumptions and constraints; and our perception of inherent risks and the potential. Schools are a collective engagement, so the action in one learning space is not isolated from those elsewhere in the school. The collaboration is within the class and across the classes, conveyed by the culture of the institution and the behaviours within. A significant challenge though is that of undertaking change. Change is imbued with risk and our reaction is in part a mitigation of this risk, a constructive engagement of the risk.

If spaces are not determinist, the design of learning environments is therefore focussed on the provision of opportunity, delivering spaces that have the potential to accommodate, satisfy or enable a variety of desired experiences.

\section{Making Informed Choices}

While I have been writing this from the perspective of the teacher rather than the student, both students and teachers react to the space in which they are engaged in learning. The chapters in this book reflect both perspectives, as well as those of school leaders. At the centre of our focus is what happens in the space at the moment of learning. Everyone in the room will respond to the task, the space and the moment. 
For some, their role is to anticipate and prepare for the task, for others it is to engage in the performance of the task.

A key purpose of the research in LEaRN is to inform our choices and to assist in change. The following chapters have been grouped loosely into four sections that reflect dimensions in which choice is made: Change \& Risk, Habitation, Measurement and Teacher Practice. The contents of papers naturally cross between the sections, so an interest in one aspect may lead you to read papers in another section. Each section is introduced with a brief overview of the papers, here you might find guidance to related topics.

The collection starts with a consideration of the change that teaching practice is undergoing when it is adapted to new environments. As I noted above, change is inherently risky, although stasis is also imbued with risk but often assumed to be risk-free. Seldom is change wholesale; instead, changes can be made across the range of factors. In our focus, it may be practices, physical features or temporal. Obviously, change must also be made in a coherent and coordinated manner, otherwise change in one aspect is obviated by lack of change elsewhere. While we observe that good teachers can teach well in almost any setting, even their effectiveness can be hobbled by poor leadership or frustrated by inadequate settings. Thus, we can conclude that some changes are positive advances, other changes might be undertaken in mitigation.

When we enter a space new to us and our intended activity, we naturally engage in some assessment of the environment. Some may do so explicitly, testing the acoustics or manipulating the lighting or changing the disposition of the furniture. Others may be less explicit in their adaptation, reacting tacitly to features and feedback. Howsoever we do this, we are inhabiting the space but also to cohabit with those around us, either the students in the room or the teachers and students in adjoining spaces. The act of habitation is a negotiation with animate and inanimate, conversing with the mutable and accommodating the immutable.

Choice must be informed, otherwise it is blind. A central driver of the research within LEaRN has been to inform our choices by providing means to measure change so that future change can be understood. The metrics must address the broad range of factors that affect outcomes of change.

Ultimately, the goal of change is to enable more effective learning and to assist teachers with these responsibilities by providing research-based insights as to what improved learning might be, how it might be supported and how new learning environments offer opportunities for such outcomes. Most immediately, therefore, the insights in the chapters will be of use to teachers in their particular contexts, complementing their portfolios of capabilities they draw upon to guide students towards good learning outcomes.

The chapters will also be of value to school leaders and education policymakers as they provide perspectives on how opportunities for change are realised provided that policies allow for the initiative. The benefits need not be driven by capital investments but where those are necessary, the interpretation for action might lead to other than prescribed. 
With the multidisciplinary team that has conducted the research, we are able also to offer insights in the design of innovative learning environments, discussing examples from several places in the world and examine their use.

While each of these aspects is in itself of importance, perhaps the most valuable consequence of the research presented here is the integration of perspectives. Wesley Imms addresses this specifically in his chapter where he writes about the interpretation and application of the research insights and how these suggest our next challenges. The summative lesson to be drawn from the work presented here is that this is a work in progress and that the field is ever-evolving.

This chapter has illustrated that guiding capital investments in schools to deliver better outcomes is a broad and multifaceted challenge. The value of the investments may be primarily intended to help students learn more effectively or more deeply, but that outcome can be affected by many factors. While that is a primary focus, the many other ways in which schools contribute to communities can also be considered. Research has an important role in illuminating this and helping us to make our choices.

\section{References}

Cuffaro, H. K. (1995). Experimenting with the world: John Dewey and the early childhood classroom. Teachers College Press.

Dewey, J. (2001). The school and society \& the child and the curriculum. NY: Mineola. United Kingdom. (1943). House of Commons. Debates: 28 October.

Thomas Kvan (Australia) is recognised for his pioneering work in design, digital environments and design management and has held senior leadership roles in several universities as Dean and Pro Vice Chancellor. He was the founding co-Director of LEaRN (the Learning Environments Applied Research Network) delivering multidisciplinary research on learning and architecture, and was founding Director of AURIN (the Australian Urban Research Information Network) that developed a national digital infrastructure, both networks hosted at The University of Melbourne. He has published over 180 publications in academic, professional and popular channels. He is currently founding Dean of the School of Design at the Southern University of Science and Technology (SUSTech) in China. 
Open Access This chapter is licensed under the terms of the Creative Commons Attribution 4.0 International License (http://creativecommons.org/licenses/by/4.0/), which permits use, sharing, adaptation, distribution and reproduction in any medium or format, as long as you give appropriate credit to the original author(s) and the source, provide a link to the Creative Commons license and indicate if changes were made.

The images or other third party material in this chapter are included in the chapter's Creative Commons license, unless indicated otherwise in a credit line to the material. If material is not included in the chapter's Creative Commons license and your intended use is not permitted by statutory regulation or exceeds the permitted use, you will need to obtain permission directly from the copyright holder.

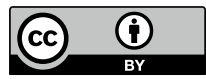

